\title{
Effective Interactions of Relativistic Composite Particles in Unified Nonlinear Spinor-Field Models. I
}

\author{
H. Stumpf \\ Institut für Theoretische Physik, Universität Tübingen
}

Z. Naturforsch. 40a, 14-28 (1985); received September 5, 1984

\begin{abstract}
Unified nonlinear spinor field models are selfregularizing quantum field theories in which all observable (elementary and non-elementary) particles are assumed to be bound states of fermionic preon fields. Due to their large masses the preons themselves are confined. In preceding papers a functional energy representation, the statistical interpretation and the dynamical equations were derived. In this paper the dynamics of composite particles is discussed. The composite particles are defined to be eigensolutions of the diagonal part of the energy representation. Corresponding calculations are in preparation, but in the present paper a suitable composite particle spectrum is assumed. It consists of preon-antipreon boson states and threepreon-fermion states with corresponding antifermions and contains bound states as well as preon scattering states. The state functional is expanded in terms of these composite particle states with inclusion of preon scattering states. The transformation of the functional energy representation of the spinor field into composite particle functional operators produces a hierarchy of effective interactions at the composite particle level, the leading terms of which are identical with the functional energy representation of a phenomenological boson-fermion coupling theory. This representation is valid as long as the processes are assumed to be below the energetic threshold for preon production or preon break-up reactions, respectively. From this it can be concluded that below the threshold the effective interactions of composite particles in a unified spinor field model lead to phenomenological coupling theories which depend in their properties on the bound state spectrum of the self-regularizing spinor theory.
\end{abstract}

PACS 11.10 Field theory

PACS 12.35 Composite Models of Particles

\section{Introduction}

Unified nonlinear spinorfield (三NSF) models are quantum field theories in which all observable (elementary and non-elementary) particles are assumed to be bound states of elementary fermion fields. Accordingly, such models must be formulated by dynamical laws for self-coupled fermion fields only.

The fundamental dynamical laws for self-coupled fermion fields are given by NSF equations with first order derivatives (三 FDNSF) and local interactions. However, if canonical quantization is applied to such equations, the corresponding quantum field theory is non-renormalizable. In spite of this nonrenormalizability in the development of quantum field theory numerous attempts were made to give these models a physical and mathematical meaning. Among these attempts are methods which introduce cut-offs, nonlocal interactions, lattice approximations

Reprint requests to Prof. Dr. H. Stumpf, Institut für Theoretische Physik der Universität Tübingen, Auf der Morgenstelle 14, D-7400 Tübingen. and other arbitrary regularization procedures. Such methods have in common that they impose ad hoc manipulations on a quantum field theory which are not contained in the theoretical basis of the theory. Therefore, their theoretical value is dubious and an approach is needed which does not make use of such manipulations.

One possibility to circumvent the non-renormalizability of FDNSF equations in a more systematic manner is the use of higher order derivative nonlinear spinorfield ( $\equiv$ HDNSF) equations. These equations exhibit self-regularizing properties and are thus renormalizable or super-renormalizable. However, in the framework of canonical quantization they imply the introduction of indefinite metric in the corresponding state space which necessitates further investigations.

Higher order derivative field equations were first introduced by Bopp [1] in constructing a selfregularizing classical electrodynamics. Later on Podolski [2] made the same proposal. Wildermuth [3] first discussed the canonical quantization of higher order derivative linear spinorfield equations. 
A survey of further development would exceed the scope of this paper. Therefore, we refer with respect to this outline to a preceding paper [4].

Using quantized HDNSF models, five different problems have to be treated and solved in order to provide a sound physical and mathematical basis for the application of such models:

i) the foundation of the self-regularizing HDNSF equation itself;

ii) the proof that in spite of the indefinite metric a meaningful physical and statistical interpretation of the theory is guaranteed;

iii) the derivation of the bound state spectrum;

iv) the proof that bound states representing "elementary particles" satisfy in certain approximations the corresponding gauge theories which govern the reactions of these particles if they are considered to be elementary and pointlike;

v) the derivation of corrections of conventional gauge theories which result from the nonelementarity of their "elementary" constituents.

Although numerous efforts were made in the past to treat NSF models, no satisfactory and systematic answers are available in the literature for the solution of these peculiar problems. Hence a systematic approach is needed to solve these problems. Concerning the topical importance of unified NSF models in elementary particle physics Grosser [5] showed that their use is unavoidable at the preon level. Therefore, a research program was started by the author and collaborators in this field. The following results have been established so far: Grosser and Lauxmann [6] proposed an NSF model with second order derivatives at the preon level. Based upon a decomposition theorem of HDNSF equations into a set of FDNSF equations proved by the author [7] and Grosser [8], a self-regularizing second order derivative NSF equation was derived from a FDNSF equation through a symmetry breaking quantization by the author [9]. The same decomposition theorem was used for the derivation of a functional energy eigenvalue equation for the calculation of relativistic bound states by Grosser, Hailer, Hornung, Lauxmann, and Stumpf [10]. For this type of equation the author [11] discussed the properties of solutions in the two-fermion sector and confirmed the possibility of giving a correct statistical interpretation of the theory. This method can easily be extended to higher fermion sectors. Thus, as far as the problems i), ii) and iii) are concerned, they are solved in principle. Naturally further investigations have to be performed in order to confirm and generalize the results obtained and to calculate the bound state spectrum numerically. Corresponding papers are in preparation. Therefore, at the present stage of the theory the problems iv) and v) need to be investigated. The treatment of both these problems is very complicated. A first step in this direction is done in this paper. Before formulating our own peculiar approach we discuss the approaches given in the literature.

If in iv) and v) the term "gauge theory" is replaced by "effective interactions" the problems iv) and v) are no longer specific for high energy physics. Rather they are always encountered where composite particles play a decisive role in physical processes. Thus, these problems are not only treated in high energy physics but also in nuclear physics, solid state physics and molecular physics. Generally all approaches can be grouped into two classes: the composite particle operator description and the composite particle state description.

Apart from numerous Bethe-Salpeter bound state calculations etc. which in the strict sense are no genuine state calculations but only projection calculations, in the literature the composite particle operator description is specific for high energy physics, while, for instance, in nuclear physics the composite particle state description is mainly used.

The introduction of composite particle operators or states does not immediately imply the derivation of effective field theories for such "clusters". Such effective field theories for "clusters" are rather a further step in the use of composite particle operators or (and) states. As we are mainly interested in this problem we do not extensively review the mere introduction of such quantities in high energy physics but concentrate on those approaches where in addition to the introduction of composite particle quantities effective field theories for "clusters" were derived.

The idea of generating relativistic composite particles, in particular bosons, by fusion of elementary relativistic fermions was inaugurated by de Broglie [12] who assumed the photon to be fusioned from two neutrinos. Later on, Fermi and Yang [13] proposed the pion to be a relativistic composite particle formed by a nucleon-antinucleon bound state. Finally, in the NSF approach of Heisenberg [14] any existing boson is assumed to be a bound 
state of elementary relativistic fermions, and of course also the complicated heavy fermions are assumed to be bound states of such elementary fermions. These authors, however, did not try to systematically establish a connection between the field laws of the elementary fermions and the effective interactions between their bound states. The first step in this direction was taken by Jouvet [15] who studied equivalence proofs between FDNSF models and boson-fermion coupling theories ( $\equiv \mathrm{BFCT}$ ) by considering the four-fermion interaction as the limit of an unrenormalized boson exchange interaction between fermionic currents where the bosons are fermionic bound states. The derivation of such an equivalence proof was motivated by the claim to make a nonrenormalizable FDNSF equation renormalizable via a map on to a renormalizable BFCT model. Another approach in this field was initiated by Haag [16], Nishijima [17] and W. Zimmermann [18] who proposed a definition of local field operators for composite particles in terms of elementary fields and an extension of the L.S.Z. S-matrix formalism to include such operators. In the further evaluation of the map between FDNSF models and BFCT models bilinear spinorfield expressions were identified with boson operators, and several authors, Bjorken [19], Bialynicki-Birula [20], Lurie and Macfarlane [21], Guralnik [22], established a map of the Green function equations of self-coupled fermion fields and those of quantum electrodynamics. Further progress was made by the application of the path integral representation of quantum field theory. From a given Lagrangian and the corresponding path integral R. L. Zimmermann [23] eliminated by functional integration appropriate field quantities and arrived at equivalent non-local Lagrangians with a reduced number of fields which for vanishing wavefunction renormalization constants of the eliminated fields become local. The latter condition coincides with the condition of Jouvet's proof. A survey of the field theoretical approach to composite particles, in particular with respect to his own method and its application to various nonrelativistic models, was given by Jouvet [24]. Another way of proving the equivalence between FDNSF equations and BFCT models was proposed by Mandelbrojt [25] who tried to establish a relation between both theories by means of a unitary transformation of the field operators, a method being similar to canonical transformations in solid state physics. Massidda and Tirapegui [26] and Esposito and Esposito [27] elaborated the consequences of Jouvet's $Z=0$ condition in more detail. Tirapegui [28] related the Haag-Nishijima-Zimmermann ( $\equiv$ HNZ) construction of composite field operators to the equivalence proof with the $Z=0$ condition. Wilson and W. Zimmermann [29] and Otterson and W. Zimmermann [30] analyzed the short distance behaviour of field operators products from an axiomatic point of view and subsequently derived a more refined version of the construction of local composite field operators. Yuk-Ming P. Lam and Schroer [31] gave a simplified proof of the equivalence theorem of Lagrangian field theories based on Gell-Mann-Low perturbation theory. Using the technique of auxiliary fields which was introduced by Coleman, Jackiw and Politzer [32] and Gross and Neveu [33], Kugo [34] and Kikkawa [35] derived by means of functional integration equivalent path integral expressions for self-coupled fermion fields and BFCT. This method was improved by Eguchi [36] and extended by several authors, Saito and Shigemoto [37], Terazawa, Chikashige, and Akama [38], Konisi, Miyata, Saito, and Shigemoto [39], to establish equivalences between fermion self-coupled theories and electro-weak and strong fermion-boson models. Similar approaches, in particular with respect to demonstrate the renormalizability of NSF models, were made by Tamvakis and Guralnik [40], Campell, Cooper, Guralnik, and Snyderman [41], Haymaker and Cooper [42], Cooper, Guralnik, Haymaker, and Tamvakis [43], Shizuya [44], Munczek and Nemirovsky [45], Rembiesa [46], Konisi and Takahashi [47], Sharatchandra [48], Chiang Chiu, Sudarshan, and Tata [49], Munczek [50], Chia, Chiu and Chou [51] etc. Thus it seems, as if the biunique map between non-renormalizable FDNSF and renormalizable FBCT is well understood at present.

Unfortunately, however, this is not the case as there still remain open problems which are concerned with the conditions under which such equivalences are proved. In all these approaches it is necessary to introduce cut-offs, infinite and vanishing renormalization constants etc. But using such procedures it is completely unclear what "being equivalent" really means. For instance, Broido [52] showed that the photon cannot be assumed to be a bound state of fermion-antifermion pairs within the framework of the $Z=0$ equivalence theorem. La 
Camera and Wataghin [53] emphasized that the $Z=0$ condition is unacceptable. The conditions of such maps were systematically discussed by Kerler [54] and reemphasized by him, Kerler [55]. In detail he investigated the drawbacks of such methods and concluded that a consistent biunique map of the above kind is impossible. This was confirmed by results of Eguchi [56] who discussed renormalization subsidiary conditions with respect to such maps. Also Tamvakis and Guralnik [57] studied such conditions and noticed that meaningful field theoretic models in four dimensional space time with nontrivial fix-points wait to be discovered. Banks and Zaks [58] showed that NSF models with local vector-condensates lead to observable violation of Lorentz invariance and not to effective gauge theories. Chiang, Chiu, Sudarshan and Tata [59] emphasized that the present equivalence proofs of NSF and BFCT models need to be reexamined as they are not sufficient to ensure equivalence. Nakanishi [60] criticized the bosonization of fermions and Ellwanger [61] stated that no exact method is available to demonstrate that fermionantifermion condensation actually takes place in a given theory. Summarizing these results, it seems that the above mentioned techniques to prove equivalences between FDNSF and BFCT models are more dangerous and misleading than promising approaches in this field.

Some of the difficulties encountered in the above approaches can be removed if selfregularizing NSF equations are used. In this case no emphasis is laid on the attempt to make nonrenormalizable NSF models via a map renormalizable. Rather one is interested to study the effective interactions of composite particles arising from bound states of selfregularizing NSF models. Recently such an approach was extensively elaborated by Dürr [62] and Saller [63] in using "noncanonical" basic spinorfields. The essential assumptions introduced by these two authors consist in the use of a Wilson expansion of local or nonlocal spinorfield operator products and the definition of gauge boson operators by the finite part of such products. Then by dressing the field operators, by assuming degenerate vacua and superconducting field configurations, by functional integration etc. they arrive at FBCT models and vice versa after appropriate rearrangements from HDNSF models. The work of both authors is interesting with respect to the reduction of the variety of "phenomenological" fields according to group theoretical and other dynamical properties of basic spinorfields. A similar approach was performed by Amati and Veneziano [64] who studied the generation of gravity theory from HDNSF models. In both of these approaches completely satisfactory solutions of the map problem between HDNSF models and FBCT models have, however, not yet been reached so far.

Apart from the technical problems, a basic objection remains which refers to all approaches discussed above, i.e. to HDNSF models as well as to FDNSF models: If gauge bosons, and in preon models even fermions, are described by products of two or more spinorfield operators they are deprived of nearly any remarkable physical content apart from their transformation properties. However, if bosons and fermions are genuine bound states of elementary fermion fields, then under suitable kinematical conditions their behaviour will differ from that of elementary pointlike particles. In particular, one has then to expect a similar richness of the physical phenomena at the high energy level as is known at the low energy level from atomic and nuclear physics. The variety of such phenomena cannot appropriately be described by operator products. Rather the wave functions, i.e. state representations, are needed, and thus the state approach to the derivation of effective interactions seems superior to the operator approach Therefore we will pursue this approach in the following.

The first attempt to describe the reactions of composite particles in quantum mechanics was made by Wheeler [65] who introduced the resonating group method. Later on, Griffin and Wheeler [66] proposed the generator coordinate method to treat collective motions in nuclei. Ekstein [67] started the theory of non-relastivistic multichannel scattering processes. Wildermuth and Kannelopoulos [68] reintroduced the resonating group approach by proposing the "cluster model" of nuclei. Wildermuth and collaborators extensively worked out this model to include all types of nuclear reactions, cf. Wildermuth and Tang [69]. Jordan [70] formulated a manifest Lorentz invariant Hamiltonian formalism for multichannel scattering processes. Koutchouk [71] constructed Fock spaces for nonrelativistic dressed particles, i.e. for bound states, and tried to discuss the effective dynamics of these particles. A similar approach was made by Girardeau [72] who 
studied a second quantization representation for systems containing bound states, in particular atoms, nuclei and electrons. Rosenberg [73] developed a theory of nonrelativistic cluster approximations. Goldberg and Puff [74] discussed the formulation of a nonrelativistic many-body theory for composite particles in terms of second quantization. Along similar lines Gilbert [75] and Kvasnicka [76] discussed the second quantization representation for nonrelativistic composite particles. Further references are contained in the papers of these authors. Kramer [77] and collaborators extensively studied the group theoretical aspects of the interactions of composite nucleon systems. In a series of papers Schmid [78] studied the nonrelativistic N-cluster dynamics and the effective interactions of composite particles and discussed the properties of the corresponding dynamical equations. The work of Wildermuth, Kramer and Schmid in particular reveals the inherent richness of composite particle theories and reactions which cannot be imitated by field operators alone.

In contrast to high energy physics in nuclear and solid state physics one is not immediately forced to detect well-known phenomenological laws which underly strict symmetry conditions etc. In addition, of course, the relativistic invariance plays no role. Thus, even if we intend to apply the composite particle state description to high energy phenomena we cannot simply copy the nonrelativistic methods. Rather we have to develop a genuine field theoretical method which is adapted to the needs of relativistic quantum field theory. The appropriate tool is functional quantum theory which was developed by the author and his collaborators. For brevity in this introduction we do not describe in detail the various steps required for an appropriate treatment of our problem but confine ourselves to discuss the essentials of the model used.

By definition of NSF models all observable particles are assumed to be theoretically described as bound states of elementary unobservable fermion fields. In our case these elementary fermion fields are given by $\varphi^{1}(x)$ and $\varphi^{2}(x)$ of Equation (1.3). According to a preceding paper [9] the masses $m_{1}$ and $m_{2}$ which appear in the corresponding equations of motion (1.2) have to be interpreted as "chemical potentials" for elementary neutrino fields. If these neutrino fields are assumed to describe the constituents of the ground state of the model, i.e. for instance, the cosmological ground state, then the chemical potentials must take very large numerical values in order to reproduce the total mass of the cosmos by the average energy of a neutrino gas. Therefore, $m_{1}$ and $m_{2}$ must have very large numerical values, too. If one now forgets about the origin of these masses and treats the model in the common interaction representation, then the "elementary fermions" which correspond to the solution of the free field equations for the $\varphi^{1}$ and $\varphi^{2}$ fields, are completely confined. Due to their large masses the energetic threshold for the production of such "elementary fermions" is so high that it could be reached only at the expense of providing the average ground state energy of the cosmos. As this will never be the case, these "elementary fermions" will remain unobservable. Thus, by this mechanism the first part of the definition of NSF models concerning their elementary constituents can be fulfilled. As far as the second part of the definition of NSF models is concerned it was shown for the special case of two-fermion bound states [11]: a consistent probability interpretation for bound states is possible and in spite of the large constituent masses there exist low energetic bound states with small masses. The procedure used for the two-fermion sector can be easily generalized to the treatment of the $n$-fermion sector where one has to expect similar positive results. Special calculations are in preparation. In order to perform further investigations as long as no detailed bound state calculations are available we therefore assume:

The NSF model (1.1) has a reasonable bound state spectrum with a consistent probability interpretation.

As the elementary fermions remain unobservable the states of the bound state spectrum are the only quantities which are related to observable results. Therefore, the whole dynamics of the model must be formulated in terms of these states. From these considerations it is obvious that it is impossible to realize this program by means of the field operators only, as these operators contain a mixture of observable and unobservable ingredients. Therefore, we are forced to use the state concept.

It was described above that the state concept has been applied mainly in nuclear physics. In nuclear physics the transition to a composite particle description is performed by a so-called "cluster expansion" where it should be noted that the notion 
"cluster expansion" is used in a different meaning in high energy physics, statistical mechanics and nuclear physics. In the following we will use the notion "cluster expansion" in the meaning of nuclear physics as an abbreviation of composite particle or bound state expansion calculations.

In order to work with states in quantum field theory functional quantum theory can be used where the ordinary states of quantum mechanics are replaced by state functionals. In terms of these state functionals a functional relativistic cluster theory was developed by the author, see for instance the early review article [79].

Later on a consistent, manifest relativistic invariant system of functional equations was derived [80] for the calculation of bound state functionals and of corresponding multichannel bound state scattering functionals, i.e. for the description of composite particle reactions. Unfortunately, this approach does not seem to allow easily to elaborate the points ii), iv) and v) in a general convincing manner. Therefore, in order to solve these problems, the functional energy representation was investigated in more detail by the author and collaborators, the results of these investigations being cited at the beginning. In using this representation as a starting point of a relativistic cluster expansion the question arises immediately whether such an expansion can be really performed without violating relativistic invariance.

In ordinary quantum theory one is confronted with the same problem by using the energy representation, i.e. in this case the Schrödinger equation. Tomonaga [81] and Schwinger [82] showed formally that the Schrödinger equation allows a correct relativistic transformation for relativistic quantum fields. Bopp [83] developed a quantum theory of relativistic point particles by using relativistic Hamiltonians. Jordan [70] constructed a Lorentz invariant multichannel scattering formalism based on Möller operators. Lam [84] related the noncovariant energy perturbation theory to a Feynman relativistic invariant graph representation. Halpern [85] connected formally Galilean invariant $S$-matrices to corresponding Lorentz invariant ones. With respect to cluster expansions Licht and Pagnamento [86] and Peres [87] considered the generalization of nonrelativistic cluster functions to relativistic ones. In particular, the latter approach needs further clarification.
Compared with these approaches in ordinary quantum theory the situation is more simple in functional quantum theory: The functional energy representation can be considered as a subsidiary condition for a fully relativistic invariant set of functional equations. If there is any doubt about relativistic invariance one can return to these equations in a unique way and demonstrate this invariance explicitly. This is discussed in Section 3. Therefore the requirement of relativistic invariance of the energy representation and the cluster expansion does not raise any new difficulties in functional quantum theory.

Since we forgo to describe the various steps required for a functional cluster expansion of the NSF model (1.1) in this introduction we finally only comment its results and raise some obvious questions: We assume an idealized and simplified bound state spectrum consisting of one scalar boson ( $\equiv$ preon-antipreon bound state) and of one spin $1 / 2$ fermion ( $\equiv$ three preon bound state) for all momenta and the corresponing antiparticles. The transformation of the functional energy representation (1.11) of the model (1.1) into composite particle functional operators produces an hierarchy of effective interactions at the composite particle level (9.1), the leading terms of which are identical with the functional energy representation (2.6) of a phenomenological boson fermion coupling theory (2.1) apart from quantization terms. Then three questions arise:

i) Can one derive correction terms to the phenomenological boson-fermion interaction which allow experimental verification?

ii) Is it possible for a suitable bound state spectrum to also derive nonabelian gauge theories and corresponding interactions?

iii) Does the bound state calculation allow to avoid infinities which occur in the phenomenological boson-fermion coupling theories?

Concerning i), correction terms follow from the hierarchy of interactions (9.1). But these correction terms are very small and cannot be expected to become experimentally verified. It has, however, to be observed that there exist other correction terms which do not appear in the present calculation. In order to simplify our calculation for a first step, antisymmetrization was not fully taken into account: Only the clusters themselves are internally antisymmetrized, but antisymmetrization between var- 
ious clusters was omitted. The inclusion of the latter would lead to exchange forces between clusters which describe the deviations from their pointlike behaviour and can be expected to be measurable. Corresponding calculations will be performed in forthcoming papers.

Concerning ii) it can be demonstrated that in the hierarchy of interactions (9.1) some terms appear which correspond to the nonlinear field terms in nonabelian gauge boson theories. These terms vanish for scalar bosons due to symmetry properties. For vector bosons such terms in general will not vanish. Hence there seems to be a possibility to also derive nonabelian gauge theories in the framework of such expansions.

Concerning iii) it will be demonstrated in a forthcoming paper that for renormalizable NSF models the calculation of bound cluster states by means of (3.1) is renormalization free, i.e., no infinite renormalization counter terms are required to treat these bound states. Furthermore, as will be discussed in Sect. 9, the quantization terms of the boson-fermion coupling theory are softened as soon as bosons and fermions are considered as composite particles, although the NSF model is properly canonically quantized. This seems to indicate that for a composite boson-fermion coupling theory no infinite renormalization constants will be needed.

Summarizing these comments it seems that this paper is to be considered as a first step towards a more profound understanding of relativistic composite particle interactions.

\section{Spinorfield Energy Representation}

Unified field models are formulated by means of self-regularizing HDNSF equations. In order to concentrate on the essential properties of such models we omit all algebraic complications which are required for the formulation of realistic models and we confine ourselves to the most simple version of a HDNSF equation which exhibits self-regularization, relativistic invariance and locality. Such an equation was derived from first principles by the author [9] and reads

$$
\begin{array}{r}
{\left[\left(-i \gamma^{\mu} \partial_{\mu}+m_{1}\right)\left(-i \gamma^{\varrho} \partial_{\varrho}+m_{2}\right)\right]_{\alpha \beta} \psi_{\beta}(x)} \\
=g V_{\alpha \beta \gamma \delta} \psi_{\beta}(x) \bar{\psi}_{\gamma}(x) \psi_{\delta}(x)
\end{array}
$$

with the scalar interaction $V_{\alpha \beta \gamma \delta}:=\delta_{\alpha \beta} \delta_{\gamma \delta}$.
In order to derive an energy representation we decompose this equation into a system of two FDNSF equations. It was proved by the author [7] and by Grosser [8] that the set of nonlinear equations, $r=1,2$

$$
\begin{aligned}
& \left(-i \gamma^{\mu} \partial_{\mu}+m_{r}\right)_{\alpha \beta} \varphi_{\beta}^{r}(x) \\
& =g \lambda_{r} V_{\alpha \beta \gamma \delta}\left[\sum_{r=1}^{2} \varphi_{\beta}^{r}(x)\right]\left[\sum_{r^{\prime}=1}^{2} \bar{\varphi}_{\gamma}^{r^{\prime}}(x)\right]\left[\sum_{r^{\prime \prime}=1}^{2} \varphi_{\delta}^{r^{\prime \prime}}(x)\right]
\end{aligned}
$$

is connected with (1.1) by a biunique map where this map is defined by the self-consistent relations

$$
\begin{aligned}
& \psi_{\alpha}(x)=\varphi_{x}^{1}(x)+\varphi_{\alpha}^{2}(x), \\
& \varphi_{\alpha}^{1}(x)=\lambda_{1}\left(-i \gamma^{\mu} \partial_{\mu}+m_{2}\right)_{\alpha \beta} \psi_{\beta}(x), \\
& \varphi_{\alpha}^{2}(x)=\lambda_{2}\left(-i \gamma^{\mu} \partial_{\mu}+m_{1}\right)_{\alpha \beta} \psi_{\beta}(x)
\end{aligned}
$$

with $\lambda_{1}:=(\Delta m)^{-1} ; \lambda_{2}=-(\Delta m)^{-1}$ and $\Delta m:=m_{2}-m_{1}$.

The quantization of this model was performed in [11] and of a similar, more complicated model in [10]. It turns out that the $\psi, \bar{\psi}$-anticommutator vanishes for equal times, and therefore all field operators in the interaction term of the corresponding Lagrangian completely anticommute. In order to obtain a forminvariant Lagrangian with respect to permutations of such anticommuting operators, at the quantum level the classical vertex $V_{\alpha \beta \gamma \delta}$ must be replaced by

$$
V_{\alpha \beta \gamma \delta}^{a}:=2 \delta_{\alpha \beta} \delta_{\gamma \delta}-2 \delta_{\alpha \delta} \delta_{\gamma \beta} .
$$

It is further convenient to introduce the transformed operators

$$
\Phi^{1}(x):=i_{1}^{-1 / 2} \varphi^{1}(x) ; \quad \Phi^{2}(x):=\left|i_{2}\right|^{-1 / 2} \varphi^{2}(x) .
$$

Then the quantum version of the model is defined by the state functionals

$$
\begin{aligned}
\left.\mathfrak{I}(j, a)\rangle=\sum_{n=1}^{\infty} \sum_{\substack{k_{1} \ldots k_{n} \\
r_{1} \ldots r_{n}}} \int \underset{r_{1}}{r_{1}}{\underset{x}{x_{1}}}_{k_{1}}, \ldots, \underset{x_{n}}{x_{n}} k_{n} \mid a\right) \\
\left.\cdot D_{n}\left(\underset{x_{1}}{x_{1}} k_{1}, \ldots, \underset{x_{n}}{x_{n}} k_{n}\right)\right\rangle \mathrm{d}^{4} x_{1} \ldots \mathrm{d}^{4} x_{n}
\end{aligned}
$$

with

$$
\begin{aligned}
\tau\left(\stackrel{r_{1}}{x_{1}} k_{1}, \ldots, \underset{\alpha_{n}}{x_{n}} k_{n} \mid a\right) \\
:=\left\langle 0\left|T \Phi_{\alpha_{1}}^{r_{1}}\left(x_{1} k_{1}\right) \ldots \Phi_{\alpha_{n}}^{r_{n}}\left(x_{n} k_{n}\right)\right| a\right\rangle,
\end{aligned}
$$

where $\left\langle D_{n}\right\rangle$ are appropriate functional basis states and where

$\Phi_{\alpha}^{r}(x, 1)=\Phi_{\alpha}^{r}(x) ; \quad \Phi_{\alpha}^{r}(x, 2):=\Phi_{\alpha}^{r}(x)$ i.e. $\quad k=1,2$.

Although the state functionals (1.6) are the basic quantities of the theory for concrete calculations it 
is necessary to introduce the transformed state functionals

with

$$
\left.\mathfrak{F}(j, a)\rangle=\exp \left[\sum_{\substack{k k^{\prime} \\ r r^{\prime}}} \int_{\alpha}^{r}(x, k) F_{\alpha \beta}^{r r^{\prime}}\left(x, k, x^{\prime}, k^{\prime}\right) j_{\beta}^{r^{\prime}}\left(x^{\prime}, k^{\prime}\right) \mathrm{d}^{4} x \mathrm{~d}^{4} x^{\prime}\right] \mathfrak{I}(j, a)\right\rangle
$$

$$
\left.\left.\mathfrak{F}(j, a)\rangle=\sum_{n=1}^{\infty} \sum_{\substack{k_{1} \ldots k_{n} \\ r_{1} \ldots r_{n}}} \int \varphi\left({\stackrel{r_{1}}{x_{1}} k_{1}}_{\alpha_{1}}, \ldots, \underset{x_{n}}{\underset{x_{n}}{x}} k_{n} \mid a\right) \mid D_{n}^{D_{x_{1}}} \underset{x_{1}}{r_{1}} k_{1}, \ldots, \underset{x_{n}}{x_{n}} k_{n}\right)\right\rangle \mathrm{d}^{4} x_{1} \ldots \mathrm{d}^{4} x_{n},
$$

where the $F$-functions in (1.9) are the free field propagators and where (1.10) can equivalently be taken as an appropriate description of the states $|a\rangle$.

The energy representation of the spinorfield, i.e. the corresponding functional equation for functional energy eigenstates of this field was discussed in [10] and for the above case given in [11]. If the index $k$ is combined with the spin index $\alpha$ to give a superspinor index $\alpha \equiv(\alpha, k)$ then the corresponding functional equation reads

$\left.E|\tilde{\mho}\rangle=\sum_{r=1}^{2} \int_{i}^{r}(\boldsymbol{r}) G_{\varepsilon \alpha}^{0}\left(i \boldsymbol{G}_{\alpha \beta} \cdot \nabla-m_{r} \delta_{\alpha \beta}\right) \partial_{\beta}^{r}(\boldsymbol{r}) \mathrm{d}^{3} r \mathfrak{F}\right\rangle$

$+g \int\left[j_{\varepsilon}^{1}(\boldsymbol{r})-j_{\varepsilon}^{2}(\boldsymbol{r})\right] G_{\varepsilon \chi}^{0} V_{\alpha \beta \gamma \delta}^{a} d_{b}(\boldsymbol{r}) d_{g}(\boldsymbol{r}) d_{\delta}(\boldsymbol{r}) \mathrm{d}^{3} \cdot r|\mathfrak{\mho}\rangle$

with

$d_{\beta}(\boldsymbol{r})=\sum_{r=1}^{2}\left[\partial_{\beta}^{r}(\boldsymbol{r})-\int F_{\beta \beta^{\prime}}^{r r^{\prime}}\left(\boldsymbol{r}, \boldsymbol{r}^{\prime}\right) j_{\beta^{\prime}}^{r^{\prime}}\left(\boldsymbol{r}^{\prime}\right) \mathrm{d}^{3} r^{\prime}\right]$

For details of the definition of all quantities in the above expression we refer to $[10,11]$ and preceding papers cited there. In particular, $\lambda_{1}$ is absorbed in $g$.

As the fields (1.5) are independent we have $F^{r r^{\prime}}=\delta_{r r^{\prime}} F^{r}$.

In order to avoid lengthy calculations it is convenient to apply a more compact notation. Taking into account $F^{r r^{\prime}}=\delta_{r r^{\prime}} F^{r}$ and considering spinor and conjugate spinorfields separately we obtain by introducing

$$
\begin{aligned}
& V_{\alpha \beta \gamma \delta}:=V\left(\underset{\alpha}{z} \underset{\beta}{\boldsymbol{x}}, \underset{\gamma}{\boldsymbol{y}},{\underset{\delta}{z}}^{\boldsymbol{z}^{\prime}}\right) \\
& \equiv g G_{\alpha \alpha^{\prime}}^{0} V_{\alpha^{\prime} \beta \gamma \delta}^{a} \delta(\boldsymbol{r}-\boldsymbol{x}) \delta(\boldsymbol{r}-\boldsymbol{y}) \delta(\boldsymbol{r}-\boldsymbol{z})(-1)^{z}
\end{aligned}
$$

and

$$
F_{\beta \beta^{\prime}}:=F\left(\underset{\beta}{\stackrel{r}{\boldsymbol{x}}, r_{\beta^{\prime}}^{\boldsymbol{x}^{\prime}}}\right)
$$

from (1.11) the equation

$$
\begin{aligned}
& E|\mathfrak{F}\rangle=j_{\alpha} K_{\alpha \beta} \partial_{\beta}|\mathfrak{F}\rangle+\vec{j}_{\alpha} \bar{K}_{\alpha \beta} \bar{\partial}_{\beta}|\mathfrak{F}\rangle \\
& \quad+V_{\alpha \beta \gamma \delta} j_{\alpha}\left[\partial_{\beta} \bar{\partial}_{\gamma} \partial_{\delta}+\bar{F}_{\gamma^{\prime}{ }^{\prime} j_{\gamma^{\prime}}} \partial_{\beta} \partial_{\delta}-F_{\delta \delta^{\prime}} \bar{j}_{\delta^{\prime}} \partial_{\beta} \bar{\partial}_{\gamma}\right. \\
& \quad-F_{\beta \beta^{\prime} j_{\beta^{\prime}}} \bar{\partial}_{\gamma} \partial_{\delta}+\bar{F}_{\gamma \gamma^{\prime}} j_{\gamma^{\prime}} F_{\delta \delta^{\prime}} \bar{j}_{\delta^{\prime}} \partial_{\beta}+F_{\beta \beta^{\prime}} \bar{j}_{\beta^{\prime}} \bar{F}_{\gamma \gamma^{\prime}} j_{\gamma^{\prime}} \partial_{\delta}
\end{aligned}
$$

$$
\begin{aligned}
& \left.-\bar{F}_{\beta \beta^{\prime}} \bar{j}_{\beta^{\prime}} F_{\delta \delta^{\prime}} \bar{j}_{\delta^{\prime}} \bar{\partial}_{\gamma}-F_{\beta \beta^{\prime}} \bar{j}_{\beta^{\prime}} \bar{F}_{\gamma \gamma^{\prime}} j_{\gamma^{\prime}} F_{\delta \delta^{\prime}} \bar{j}_{\delta^{\prime}}\right]|\mathfrak{F}\rangle \\
& +V_{\alpha \beta \gamma \delta} \bar{j}_{\alpha}\left[\bar{\partial}_{\beta} \partial_{\gamma} \bar{\partial}_{\delta}+F_{\gamma \gamma^{\prime}} \bar{j}_{\gamma^{\prime}} \bar{\partial}_{\beta} \bar{\partial}_{\delta}-\bar{F}_{\delta \delta^{\prime}} j_{\delta^{\prime}} \bar{\partial}_{\beta} \partial_{\gamma}\right. \\
& -\bar{F}_{\beta \beta^{\prime}} j_{\beta^{\prime}} \partial_{\gamma} \bar{\partial}_{\delta}+F_{\gamma \gamma^{\prime}} \bar{j}_{\gamma^{\prime}} \bar{F}_{\delta \delta^{\prime}} j_{\delta^{\prime}} \bar{\partial}_{\beta}+\bar{F}_{\beta \beta^{\prime}} j_{\beta^{\prime}} F_{\gamma \gamma^{\prime}} \bar{j}_{\gamma^{\prime}} \bar{\partial}_{\delta} \\
& \left.-\bar{F}_{\beta \beta^{\prime}} j_{\beta^{\prime}} \bar{F}_{\delta \delta^{\prime}} j_{\delta^{\prime}} \partial_{\gamma}-\bar{F}_{\beta \beta^{\prime}} j_{\beta^{\prime}} F_{\gamma \gamma^{\prime}} \bar{j}_{\gamma^{\prime}} \bar{F}_{\delta \delta^{\prime}} j_{\delta^{\prime}}\right]|\mathfrak{F}\rangle \\
& :=\mathscr{H}[j, \bar{j}, \partial, \bar{\partial}]|\mathfrak{F}\rangle,
\end{aligned}
$$

where the summation convention is used throughout and where normal ordering is assumed in the interaction term of the original quantum field equation. The indices $r, r^{\prime}, r^{\prime \prime}$ are intentionally omitted at the right-hand side of (1.13) as they correspond to the free summation which appears in the interaction term of Equation (1.2). The operators $K_{\alpha \beta}$ and $\bar{K}_{\alpha \beta}$ are symbols for the kinematic terms appearing in (1.11) and need no further explanation.

The Eq. (1.15) is the exact functional equation for the $\mathfrak{F}$-functional state resulting through a direct derivation from the original field equation without any approximation or manipulation. It will be the starting-point of our further considerations. Only in the ultimate stages of calculation we will return to the more detailed indexing used before.

\section{Coupling Theory Energy Representation}

The simplest model of a coupling theory is realized by the interaction of spin $1 / 2$ fermions with scalar mesons. Its phenomenological field equations read

$$
\begin{aligned}
& \left(-i \gamma^{\mu} \partial_{\mu}+m\right)_{\alpha \beta} \chi_{\beta}(x)=g \vartheta(x) \chi_{\alpha}(x), \\
& \left(\square-\mu^{2}\right) \vartheta(x)=-g \chi_{\alpha}(x) \bar{\chi}_{\alpha}(x),
\end{aligned}
$$

where $\chi$ means the phenomenological fermion field and $\vartheta(x)$ the boson field. For a comparison with the dynamics of the unified NSF model we need the functional energy representation for this model, too. In order to derive this representation we introduce 
the conjugate momentum $\Pi$ of the $\vartheta$-field. This yields the equations

$$
\begin{gathered}
\left(-i \gamma^{\mu} \partial_{\mu}+m\right)_{\alpha \beta} \chi_{\beta}(x)=g \vartheta(x) \chi_{\alpha}(x), \\
\frac{\partial}{\partial t} \vartheta(x)=\Pi(x) \\
-\frac{\partial}{\partial t} \Pi(x)+\left(\Delta-\mu^{2}\right) \vartheta(x)=-g \chi_{\alpha}(x) \bar{\chi}_{\alpha}(x) .
\end{gathered}
$$

The quantum version of this theory is defined by state functionals $\mathfrak{I}(\eta, \bar{\eta}, J, K, a)\rangle$ in which $\eta_{x}(x)$, $\bar{\eta}_{x}(x), J(x), K(x)$ are the sources of the $\chi, \bar{\chi}, \vartheta$ or $\Pi$ fields, respectively. The definition of state functionals and the derivation of their corresponding functional equations are given for instance by Lurie [88]. If we apply the method of [10] to these equations we obtain the following functional equation for the energy representation

$$
\begin{aligned}
& -\left[\int \eta(\boldsymbol{r})\left(i \gamma^{0} \gamma \cdot \nabla+\gamma^{0} m\right) \frac{\delta}{\delta \eta(\boldsymbol{r})} \mathrm{d}^{3} r+\int \bar{\eta}(\boldsymbol{r})\left(i \gamma^{0} \gamma \cdot \nabla-\gamma^{0} m\right) \frac{\delta}{\delta \bar{\eta}(\boldsymbol{r})} \mathrm{d}^{3} r\right. \\
& \left.\left.-\int J(\boldsymbol{r}) \frac{\delta}{\delta K(\boldsymbol{r})} \mathrm{d}^{3} r+\int K(\boldsymbol{r})\left(\Delta-\mu^{2}\right) \frac{\delta}{\delta J(\boldsymbol{r})} \mathrm{d}^{3} r\right] \mathfrak{I}\right\rangle \\
& \left.-\left[g \int \bar{\eta}(\boldsymbol{r}) \gamma^{0} \frac{\delta}{\delta \bar{\eta}(\boldsymbol{r})} \frac{\delta}{\delta J(\boldsymbol{r})} \mathrm{d}^{3} r+g \int \eta(\boldsymbol{r}) \gamma^{0} \frac{\delta}{\delta \eta(\boldsymbol{r})} \frac{\delta}{\delta J(\boldsymbol{r})} \mathrm{d}^{3} r+g \int K(\boldsymbol{r}) \frac{\delta}{\delta \eta(\boldsymbol{r})} \frac{\delta}{\delta \bar{\eta}(\boldsymbol{r})} \mathrm{d}^{3} r\right] \mathfrak{I}\right\rangle \\
& \left.+i\left[2 \int \bar{\eta}(\boldsymbol{r}) \gamma^{0} \eta(\boldsymbol{r}) \mathrm{d}^{3} r-\int K(\boldsymbol{r}) J(\boldsymbol{r}) \mathrm{d}^{3} r\right]|\mathfrak{I}\rangle=E \mathfrak{I}\right\rangle .=E|\mathfrak{I}\rangle .
\end{aligned}
$$

In this equation the first bracket contains the kinetic energy operators of the free fermions and mesons. The second bracket contains the interaction energy operators of fermions and mesons and the last bracket gives the quantization terms of both fields. While the fermion part is formulated in the original field (source) and its Hermitian conjugate field (source) or its adjungate field, respectively, this is not the case for the meson field (source). For the further use it is of advantage to also express the meson part in terms of a field and its Hermitian conjugate field. This can be done by performing a Fourier transformation of the sources in (2.3) with $\eta, \bar{\eta}, J, K$ going over into $\tilde{\eta}, \tilde{\bar{\eta}}, \tilde{J}, \tilde{K}$ and by applying the transformation

$$
\tilde{K}(\boldsymbol{p})=2^{-1 / 2} \omega(\boldsymbol{p})^{-1 / 2}\left[b(\boldsymbol{p})-b^{+}(-\boldsymbol{p})\right], \quad \tilde{J}(\boldsymbol{p})=2^{-1 / 2} \omega(\boldsymbol{p})^{1 / 2}\left[b(\boldsymbol{p})+b^{+}(-\boldsymbol{p})\right]
$$

afterwards. This transformation induces the following transformation for the functional derivatives

$$
\frac{\delta}{\delta \tilde{K}(\boldsymbol{p})}=2^{-1 / 2} \omega(\boldsymbol{p})^{1 / 2}\left[\frac{\delta}{\delta b(\boldsymbol{p})}-\frac{\delta}{\delta b^{+}(-\boldsymbol{p})}\right], \quad \frac{\delta}{\delta \tilde{J}(\boldsymbol{p})}=2^{-1 / 2} \omega(\boldsymbol{p})^{-1 / 2}\left[\frac{\delta}{\delta b(\boldsymbol{p})}+\frac{\delta}{\delta b^{+}(-\boldsymbol{p})}\right] .
$$

By performing these operations we can replace (2.3) by the following equation

$$
\begin{aligned}
E|\mathfrak{I}\rangle= & {\left[\int \eta(\boldsymbol{p}) E_{l}(\boldsymbol{p}) \frac{\delta}{\delta \eta(\boldsymbol{p})} \mathrm{d}^{3} p-\int \bar{\eta}(\boldsymbol{p}) E_{\bar{l}}(\boldsymbol{p}) \frac{\delta}{\delta \bar{\eta}(\boldsymbol{p})} \mathrm{d}^{3} p\right.} \\
& \left.+\int b(\boldsymbol{p}) E_{b}(\boldsymbol{p}) \frac{\delta}{\delta b(\boldsymbol{p})} \mathrm{d}^{3} p-\int b^{+}(-\boldsymbol{p}) E_{b}(\boldsymbol{p}) \frac{\delta}{\delta b^{+}(-\boldsymbol{p})} \mathrm{d}^{3} p\right]|\mathfrak{T}\rangle \\
& -\left\{g \int \eta(\boldsymbol{p}) \gamma^{0} \frac{\delta}{\delta \eta(\boldsymbol{s})} 2^{-1 / 2} \omega^{-1 / 2}(\boldsymbol{p})\left[\frac{\delta}{\delta b(\boldsymbol{q})}+\frac{\delta}{\delta b^{+}(-\boldsymbol{q})}\right] \delta(\boldsymbol{p}-\boldsymbol{q}+\boldsymbol{s}) \mathrm{d}^{3} p \mathrm{~d}^{3} q \mathrm{~d}^{3} s\right. \\
& +g \int \bar{\eta}(\boldsymbol{p}) \gamma^{0} \frac{\delta}{\delta \bar{\eta}(\boldsymbol{s})} 2^{-1 / 2} \omega^{-1 / 2}(\boldsymbol{q})\left[\frac{\delta}{\delta b(\boldsymbol{q})}+\frac{\delta}{\delta b^{+}(-\boldsymbol{q})}\right] \delta(\boldsymbol{p}+\boldsymbol{q}+\boldsymbol{s}) \mathrm{d}^{3} p \mathrm{~d}^{3} q \mathrm{~d}^{3} s \\
& \left.+g \int \frac{\delta}{\delta \eta(\boldsymbol{q})} \frac{\delta}{\delta \bar{\eta}(\boldsymbol{s})} 2^{-1 / 2} \omega^{-1 / 2}(\boldsymbol{p})\left[b(\boldsymbol{p})-b^{+}(-\boldsymbol{p})\right] \delta(-\boldsymbol{p}+\boldsymbol{q}-\boldsymbol{s}) \mathrm{d}^{3} p \mathrm{~d}^{3} q \mathrm{~d}^{3} s\right\}|\mathfrak{T}\rangle \\
& \left.+i\left[2 \int \bar{\eta}(\boldsymbol{p}) \eta(\boldsymbol{p}) \mathrm{d}^{3} p-\frac{1}{2} \int b(\boldsymbol{p}) b(\boldsymbol{p}) \mathrm{d}^{3} p+\frac{1}{2} \int b^{+}(\boldsymbol{p}) b^{+}(\boldsymbol{p}) \mathrm{d}^{3} p\right] \mathfrak{T}\right\rangle
\end{aligned}
$$

where for brevity the tilde notation has been suppressed. 
The energy expressions $E_{l}(\boldsymbol{p})$ and $E_{\bar{l}}(\boldsymbol{p})$ are still not diagonal in the spin space but this does not play any role for our further treatment. In order to realize the similarities between ordinary field theory and functional field theory it should be noted that the sources play the role of creation operators while the functional derivatives have to be identified with destruction operators. On the other hand, it has to be emphasized that this identification does not imply the use of the interaction picture. Due to its construction it is rather representation free as these operators are referred to the functional state space an not to the genuine state space of the quantum field under consideration.

\section{Cluster Expansion of Functional States}

In order to perform a cluster expansion, the cluster states have to be defined. For the calculation of such states in [11] the truncated functional equation

$$
\begin{array}{r}
E \mathfrak{F}\rangle=\sum_{r=1}^{2} \int j_{\varepsilon}^{r}(\boldsymbol{r}) G_{\varepsilon \alpha}^{0}\left(i \boldsymbol{G}_{\alpha \beta} \cdot \nabla-m \delta_{\alpha \beta}\right) \partial_{\beta}^{r}(\boldsymbol{r}) \mathrm{d}^{3} r|\mathfrak{F}\rangle \\
+g \int\left[j_{\varepsilon}^{1}(\boldsymbol{r})-j_{\varepsilon}^{2}(\boldsymbol{r})\right] G_{\varepsilon \alpha}^{0} W_{\alpha \beta \gamma \delta}\left[\sum_{r=1}^{2} F_{\beta \beta^{\prime}}^{r}\left(\boldsymbol{r}-\boldsymbol{r}^{\prime}\right) j_{\beta^{\prime}}^{r}\left(\boldsymbol{r}^{\prime}\right)\right] \\
\cdot\left[\sum_{r^{\prime} r^{\prime \prime}=1}^{2} \partial_{\gamma}^{r^{\prime}}(\boldsymbol{r}) \partial_{\delta}^{r^{\prime \prime}}(\boldsymbol{r})\right]|\mathfrak{F}\rangle \mathrm{d}^{3} r \mathrm{~d}^{3} r^{\prime}
\end{array}
$$

was used with $W_{\alpha \beta \gamma \delta}=V_{\alpha \beta \gamma \delta}^{a}-V_{\alpha \gamma \beta \delta}^{a}+V_{\alpha \gamma \delta \beta}^{a}$. In accordance with [11] we therefore introduce the following definition:

Definition 1: A relativistic cluster state (composite particle state) is a solution of the functional equation (3.1).

A comparison with (1.11) shows that by the truncation the functional nondiagonal elements with respect to the number of coordinates are omitted. This means that in (3.1) the effects of the polarization cloud are suppressed, i.e. (3.1) gives the "hard core" of the various clusters without further dressing these particles. The omission of this polarization cloud does not mean, however, that we are working with an approximation. Rather the polarization cloud is included at a later stage of the calculation in order to allow a successful map of HDNSF models on to BFCT models.
In the introduction the question of relativistic invariance of cluster expansions has been raised. As such expansions are formed from direct products of single cluster states the relativistic invariance of a cluster expansion is guaranteed, provided each cluster functional transforms itself correctly. This can be seen as follows: According to [10] the truncated functional equation (3.1) arises by a limiting procedure to equal time variables from the combination of the general (truncated) functional equation

$$
\begin{aligned}
\left(i G_{\alpha \beta}^{\mu} \partial_{\mu}-m_{r} \delta_{\alpha \beta}\right) \partial_{\beta}^{r}(x)|\mathfrak{\mho}\rangle & \\
= & g W_{\alpha \beta \gamma \delta}\left[\sum_{r=1}^{2} \int F_{\beta \beta^{\prime}}^{r}\left(x-x^{\prime}\right) j_{\beta^{\prime}}^{r}\left(x^{\prime}\right) \mathrm{d}^{4} x^{\prime}\right] \\
& \left.\cdot\left[\sum_{r^{\prime} r^{\prime \prime}=1}^{2} \partial_{\gamma}^{r^{\prime}}(x) \partial_{\delta}^{r^{\prime \prime}}(x)\right] \mathfrak{F}\right\rangle
\end{aligned}
$$

and the eigenvalue equation for $P_{0}$ which comes from the set of equations

$$
\int j_{\varepsilon}^{r}(x) i \partial_{\mu} \partial_{\varepsilon}^{r}(x) \mathrm{d}^{4} x|\mathfrak{F}\rangle=p_{\mu}|\mathfrak{F}\rangle .
$$

Then, owing to this procedure, any solution of (3.2) and (3.3) must also be a solution of (3.1) as the derivation of (3.1) from (3.2) and (3.3) is valid for state functionals without any restriction. Solutions of (3.2) and (3.3) are assumed to exist, as (3.2) and (3.3) are compatible. The reverse however, is also true: to any solution of (3.1) and the spacelike momentum conditions of (3.3) there exists a unique solution of (3.2) and (3.3). This can be verified by observing that (3.2) defines a Cauchy problem if the initial data for equal times, i.e. the solutions of (3.1) are given. In this case (3.2) can be considered as multitime evolution equations for which under the appropriate definition of initial data unique solutions can be found. Thus the following theorem holds:

Theorem 1: The solutions of (3.2) and (3.3) possess a biunique correspondence to the solutions of (3.1) and (3.3) momentum conditions.

It can easily be verified that the momentum conditions of (3.3) and equations (3.1) are compatible. Therefore, if necessary, any one-time cluster state functional can be uniquely continued into the corresponding multitime state functional for demonstrating relativistic invariance. 
Cluster expansions are always connected with projection operations, i.e. scalar products of states or state functionals. Therefore, in order to obtain a relativistic invariant cluster theory not only the state functionals themselves, but also the scalar products have to be relativistic invariant. If such scalar products are defined they must be compatible with the general quantum theoretical formalism. In [11] we showed that in indefinite metric spaces of the kind which occur in our model the scalar product of energy eigenfunctionals can be directly derived from the general quantum theoretical formalism. In particular, we obtained for the scalar product of two states $|p\rangle$ and $\left.p^{\prime}\right\rangle$ which correspond to solutions of the energy eigenvalue equation (3.1) and the momentum conditions (3.3) the following expression

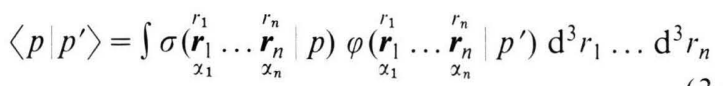

where $\sigma(\ldots \mid p)$ and $\varphi\left(\ldots \mid p^{\prime}\right)$ are the corresponding left-hand and right-hand solutions of these operators. This norm expression seems to be completely noninvariant against relativistic transformations. However, the following theorem holds:

Theorem 2: Let $|p\rangle$ and $\left|p^{\prime}\right\rangle$ be two states which are defined by their functional maps being solutions of (3.1) and the momentum conditions (3.3). Then their scalar product is given by the relativistic invariant expression

$$
\begin{aligned}
& \left.\left\langle p \mid p^{\prime}\right\rangle=\int \sigma \underset{x_{1}}{\left(x_{1}\right.} \ldots{\underset{x}{x_{n}}}_{r_{n}}^{r_{n}} p\right) \delta\left(n x_{1}\right) \ldots \\
& \left.\ldots \delta\left(n x_{n}\right) \varphi \underset{x_{n}}{\left(r_{1}\right.} \ldots{\underset{x}{x_{n}}}_{x_{n}}^{r_{n}} p^{\prime}\right) \mathrm{d}^{4} x_{1} \ldots \mathrm{d}^{4} x_{n},
\end{aligned}
$$

where $n$ is an arbitrary timelike vector.

Proof: Owing to Theorem 1 we can uniquely continue $\sigma\left(\boldsymbol{r}_{1} \ldots \boldsymbol{r}_{n} \mid p\right)$ and $\varphi\left(\boldsymbol{r}_{1} \ldots \boldsymbol{r}_{n} \mid p^{\prime}\right)$ into the multitime region to obtain $\sigma\left(x_{1} \ldots x_{n} \mid p\right)$ and $\varphi\left(x_{1} \ldots x_{n} p^{\prime}\right)$. Therefore, the integral in (3.5) is well defined. We now compare this expression for two different $n$-vectors. We have

$$
\begin{aligned}
& \left\langle p \mid p^{\prime}\right\rangle_{n}=\int \sigma_{p} \delta(n x) \varphi_{p^{\prime}} \mathrm{d} \tau, \\
& \left\langle p \mid p^{\prime}\right\rangle_{n^{\prime}}=\int \sigma_{p} \delta\left(n^{\prime} x\right) \varphi_{p^{\prime}} \mathrm{d} \tau
\end{aligned}
$$

by definition. As both the $n$-vectors are timelike we can find a Lorentz transformation $n^{\prime}=L n$ which transforms $n$ into $n^{\prime}$. We now apply this transforma- tion to the coordinates $x$, too, with $x^{\prime}=L^{-1} x$. Under this transformation we have $n^{\prime} x=n x^{\prime}$ and obtain

$$
\begin{aligned}
\left\langle p p^{\prime}\right\rangle_{n^{\prime}}= & \int \sigma\left(x_{1}^{\prime} \ldots x_{n}^{\prime} \mid L^{-1} p\right) \\
& \cdot \delta\left(n x^{\prime}\right) \varphi\left(x_{1}^{\prime} \ldots x_{n}^{\prime} \mid L^{-1} p^{\prime}\right) \mathrm{d}^{4} x_{1}^{\prime} \ldots \mathrm{d}^{4} x_{n}^{\prime} \\
= & \int \sigma\left(x_{1} \ldots x_{n} \mid L^{-1} p\right) \\
& \cdot \delta(n x) \varphi\left(x_{1} \ldots x_{n} \mid L^{-1} p^{\prime}\right) \mathrm{d}^{4} x_{1} \ldots \mathrm{d}^{4} x_{n} \\
= & \left\langle L^{-1} p L^{-1} p^{\prime}\right\rangle_{n} .
\end{aligned}
$$

Now if the functional map of the states $|p\rangle$ and $\left|p^{\prime}\right\rangle$ transforms correctly, the original states must exhibit the correct transformation property, too. Therefore, we have $\left|L^{-1} p^{\prime}\right\rangle=U\left(L^{-1}\right)\left|p^{\prime}\right\rangle$ and $\left\langle L^{-1} p\right|=$ $\left\langle p U^{-1}\left(L^{-1}\right)\right.$. Substitution of these relations in (3.7) finally gives $\left\langle p \mid p^{\prime}\right\rangle_{n^{\prime}}=\left\langle p \mid p^{\prime}\right\rangle_{n}$. Q.E.D.

As $n$ is arbitrary we can always choose it to be $n=(0,0,0,1)$ and then obtain (3.4) which is therefore equivalent to a relativistic invariant expression.

According to Definition 1 cluster expansions depend on the manifold of solutions of (3.1) and due to the required relativistic invariance according to Theorem 1 on the $\mu=1,2,3$ momentum conditions of (3.3). A short inspection of (3.1) shows that it is not necessary to take into account the $\mu=1,2,3$ momentum conditions of (3.3) explicitly. The manifold of solutions satisfying these conditions is automatically produced by (3.1) if the solutions are required to be basis states of representations of the spatial translation groups. Hence the spectrum of (3.1) alone determines the cluster expansion completely. Furthermore, it will be shown in the following that such an expansion in turn determines together with the basic equation (1.11) the map of HDNSF models on BFCT models completely. Thus, if a HDNSF model works correctly, by its spectrum that BFCT model should be produced or selected respectively which describes the phenomenological reactions of clusters in complete agreement with experiment. It is obvious that such an aim needs a large number of iteration steps for its realization.

At the present stage of the investigation no detailed knowledge about the manifold of solutions of (3.1) is available. Therefore we are forced to make plausible assumptions about this manifold in order to work with it, and it is reasonable that we confine ourselves to assumptions about the spec- 
trum which lead to the most simple but realistic BFCT models. Such a model with spin $1 / 2$ fermions and antifermions and scalar mesons was discussed in Section 2. As the elementary fermions of our HDNSF model are energetically confined, the observable fermions must be bound states of these elementary fermions. The lowest number of elementary fermions which is needed for the construction of a spin $1 / 2$ cluster is three. We thus postulate:

Assumption 1: The set of solutions of (3.1) contains only one subset of bound state solutions which are given by the two-fermion and three-fermion bound states

$$
\begin{aligned}
& \left\{\varphi_{2}\left(\boldsymbol{r}_{1}, \boldsymbol{r}_{2} \mid \boldsymbol{k}\right) \forall \boldsymbol{k} \in R^{3\}},\right. \\
& \left\{\varphi_{3}\left(\boldsymbol{r}_{1}, \boldsymbol{r}_{2}, \boldsymbol{r}_{3} \mid \boldsymbol{k}\right), \bar{\varphi}_{3}\left(\boldsymbol{r}_{1}, \boldsymbol{r}_{2}, \boldsymbol{r}_{3} \mid \boldsymbol{k}\right) \forall \boldsymbol{k} \in R^{3}\right\},
\end{aligned}
$$

where $\boldsymbol{k}$ is the center of mass momentum and where $\varphi_{2}$ represents a scalar boson, while $\varphi_{3}$ and $\bar{\varphi}_{3}$ are spin $1 / 2$ fermions and antifermions. For the corresponding masses $m_{b}$ and $m_{l}$ holds $m_{b}, m_{l} \ll m_{1}, m_{2}$.

In particular, Assumption 1 implies that the scalar particle is identical with its antiparticle while for the spin $1 / 2$ cluster-fermion particles and antiparticles are different.

As was shown in [11] for the two-fermion case, in the limit of large $m_{1}$ and $m_{2}$ masses the operator of (3.1) practically becomes Hermitian. If we extrapolate this result to higher sectors which are defined by the number of elementary fermions $n=3,4, \ldots$, we have to expect that in any sector a complete set of solutions must exist. On the other hand, apart from the bound states of Assumption 1 according to this assumption, no further bound states can exist. Thus, in these and in all other sectors the remaining solutions being required by the completeness condition must be scattering states. As the rest masses of the bound states are small, in practically all energy ranges scattering processes of bound states may occur. In contrast, the scattering processes of elementary fermions alone or of bound states with participation of elementary fermions occur only above the energetic threshold of elementary fermion production which is very high owing to their large masses. Thus, below the energetic threshold of elementary fermion production only bound states and bound state scattering occur and the theory looks like a theory of these particles only. It is this region where we shall map the HDNSF model on to a BFCT model. This leads to the assumption
Assumption 1a: The model will be treated below the threshold of elementary fermion production.

This assumption, however, does not mean that we have to introduce a cut-off. Rather the theory is at any state relativistic invariant. The only thing that happens is that above this threshold new channels are openend and therefore, due to the appearance of elementary fermions the map of HDNSF to BFCT breaks down. This break-down of the map is accompanied by the appearance of indefinite metric. While the bound states have a positive norm according to [11], the elementary fermions partly have a negative norm and so their scattering states. Therefore, above this threshold where elementary fermions are allowed to occur the statistical interpretation breaks down. This means:

HDNSF models possess a high energy limit of their applicability which manifests itself not by a violation of relativistic invariance, but by the breakdown of the statistical interpretation.

This seems to be a very interesting property as we never loose relativistic invariance, though still avoiding the dangerous claim to have a theory valid for all energy ranges. Thus in the following we will work only in the physically meaningful channels below the break-up where all dangerous elementary fermion states are hidden states. In the first step of our discussion we will, however, formally include hidden states, too, as they play an important role in transforming state functionals of our HDNSF model onto to cluster functional states of BFCT models.

The state functionals of HDNSF models as well as of BFCT models are defined by expansions with respect to basis functional states which in their turn arise from functional source operators by a Fock space construction. Having in view a map between both kinds of models in terms of functional states and equations we must first establish a connection between the source operators of these basis functionals.

Returning to the simplifying notation of Sect. 1 we denote the two-particle solutions of (3.1) for elementary fermion number $\mathscr{N}=0$ by $C_{n}^{u r}$ and the three-particle solutions for elementary fermion number $\mathscr{N}=3$ and $\mathscr{N}=-3$ by $C_{q}^{r s t}$ and $\bar{C}_{w}^{a b c}$ respectively. The complete sets of these solutions $\left\{C_{n}^{u v}\right\}$, $\left\{C_{q}^{r s t}\right\},\left\{\bar{C}_{w}^{a b c}\right\}$ then contain not only the physically meaningful bound state solutions but also the unphysical scattering solutions of elementary fermions. 
We now define the cluster source operators

$$
\begin{aligned}
& b_{n}:=C_{n}^{u r} j_{u} \overline{j_{l}}, \\
& l_{q}:=C_{q}^{r s t} j_{r} j_{s} j_{t}, \\
& \bar{l}_{w}:=\bar{C}_{w}^{a b c} \overline{j_{a}} \overline{j_{b}} \overline{j_{c}},
\end{aligned}
$$

where here and in the following the summation convention is used. Then the following theorem holds:

Theorem 3: Between the cluster source operators (3.8), (3.9a) and (3.9b) and the corresponding products of fermion source operator a biunique map exists.

Proof: As the solution of (3.1) $\left\{C_{n}^{u v}\right\},\left\{C_{q}^{r s t}\right\}$, $\left\{\bar{C}_{w}^{a b c}\right\}$ are assumed to form complete sets in their corresponding state spaces, there must exist corresponding dual state sets $\left\{R_{n}^{u v}\right\},\left\{R_{q}^{r s t}\right\},\left\{\bar{R}_{w}^{a b c}\right\}$ such that the orthonormality relations

$$
\begin{aligned}
& R_{n^{\prime}}^{u r} C_{n}^{u r}=\delta_{n^{\prime} n}, \\
& R_{q^{\prime}}^{r s t} C_{q}^{r s t}=\delta_{q^{\prime} q}, \\
& \bar{R}_{w^{\prime}}^{a b c} \bar{C}_{w}^{a b c}=\delta_{w^{\prime} w},
\end{aligned}
$$

and the completeness relations

$$
\begin{aligned}
& \sum_{n} R_{n}^{u^{\prime} r^{\prime}} C_{n}^{u r}=\delta_{u^{\prime} u} \delta_{v^{\prime} r}, \\
& \sum_{q} R_{q}^{r^{\prime} s^{\prime} t^{\prime}} C_{q}^{r s t}=\delta_{r^{\prime} r} \delta_{s^{\prime} s} d_{t^{\prime} t}, \\
& \sum_{w} \bar{R}_{w^{\prime} b^{\prime} c^{\prime}}^{a^{\prime}} \bar{C}_{w}^{a b c}=\delta_{a^{\prime} a} \delta_{b^{\prime} b} \delta_{c^{\prime} c}
\end{aligned}
$$

are simultaneously satisfied, where for brevity we omitted antisymmetrization. From these relations it follows that

$$
\begin{aligned}
& j_{u} \bar{j}_{r}=\sum_{n} R_{n}^{u r} b_{n}, \\
& j_{r} j_{s} j_{t}=\sum_{q} R_{q}^{r s t} l_{q}, \\
& \bar{j}_{a} \bar{j}_{b} \bar{j}_{c}=\sum_{w} \bar{R}_{w}^{a b c} \bar{l}_{w},
\end{aligned}
$$

i.e. the inversion of (3.8), (3.9a), (3.9 b). Q.E.D.

It has to be noted that in our model the dual sets cannot be identified with the left-hand solutions of (3.1), as the scalar products $(3.10),(3.11 \mathrm{a}),(3.11 \mathrm{~b})$, of the dual set states with the right-hand solutions of (3.1) differ from the values of the physical scalar products. Hence any dual set needs a special construction. We sketch this for the case of the boson states.

According to Assumption 1 there exist boson states whose transformation properties are iso- morphic to those of pointlike scalar boson states. We therefore conclude that the spectrum of the extended bosons (and elementary fermion scattering states with the same transformation properties) must be homomorphic to those of the pointlike bosons. In particular, we expect and assume that for the extended bosons positive and negative energies occur pairwise as this is the case for pointlike bosons. The general quantum number $n$ of the boson clusters (3.8) can then be decomposed into a positive class and a negative class for all threemomenta $\{\boldsymbol{k}\}$ of the center of mass motion and for all internal quantum numbers $\{v\}$. Thus we write $\{n\} \equiv\{( \pm, \boldsymbol{k}, v)\}$. In particular for $v=0$ we obtain the bound states, while with $v \neq 0$ elementary fermion scattering states are denoted. In analogy to the pointlike boson states we assume that for the extended boson states $C^{u v}(+, \boldsymbol{k}, 0) \equiv C_{0}^{u v}(\boldsymbol{k})^{+}$and $C^{u v}(-, \boldsymbol{k}, 0) \equiv C_{0}^{u v}(\boldsymbol{k})^{-}$belongs to positive and negative energies respectively. Thus from (3.8) we obtain two sets of boson operators $\{b(+, \boldsymbol{k}, 0)\} \equiv$ $\left\{b^{(+)}(\boldsymbol{k})\right\}$ and $\{b(-, \boldsymbol{k}, 0)\} \equiv\left\{b^{(-)}(\boldsymbol{k})\right\}$ which are needed for the functional description of the bound boson state. In conventional quantum field theory the destruction and creation operators $a(\boldsymbol{k}), a^{+}(\boldsymbol{k})$ correspond to these source operators. The construction of a dual set of states for pointlike bosons was described in detail by Hagedorn [89]. If within a certain approximation the center of mass motion is separated from the internal motion of the cluster, the construction of a dual set for pointlike bosons can immediately be taken over to the extended case. We postpone this discussion to Sect. 7 where we will also give explicit formulae.

We now turn to the representation of state functionals of our HDNSF model in terms of cluster functional operators $(3.8),(3.9 \mathrm{a}),(3.9 \mathrm{~b})$. As is known from nonrelativistic nuclear physics etc. any complete cluster expansion needs an antisymmetrization with respect to all elementary fermion coordinates which occur in the various clusters. At the physical level this complete antisymmetrization leads to exchange forces which are important for many physical phenomena. On the other hand, all calculations become considerably more complicated. In a first step of discussing maps of HDNSF models upon BFCT models we therefore do without antisymmetrization, i.e. we work with a Hartree-like expansion and not with a Hartree-Fock-like expansion. 
In our abbreviated notation the functional states (1.10) can be written

$$
\mathfrak{F}\rangle=\sum_{n=1}^{\infty} \varphi_{n}\left(\alpha_{1} \ldots \alpha_{n} \mid a\right) \frac{1}{n !} j_{\alpha_{1}} \ldots j_{\alpha_{n}}|0\rangle
$$

if additionally superspinor indices are included in this compact notation. We now assume that we consider states below the energetic threshold of elementary fermion production. Then all states can be constructed by mixtures of bound cluster states only. It must therefore be possible to express this property in terms of a cluster expansion of $|\mathfrak{F}\rangle$, i.e. we get for $\mathfrak{F}\rangle$ the equivalent representation

$$
\begin{aligned}
\mathfrak{F}\rangle= & \sum_{N, K, L} c\left(n_{1} \ldots n_{N}, q_{1} \ldots q_{K}, w_{1} \ldots w_{L} \mid a\right) \\
& \cdot b_{n_{1}} \ldots b_{n_{N}} l_{q_{1}} \ldots l_{q_{K}} \bar{l}_{w_{1}} \ldots \bar{l}_{w_{L}}|0\rangle .
\end{aligned}
$$

If the cluster operators (3.8), (3.9a), (3.9b) are expressed by superspinors

$$
\begin{aligned}
& b_{n}=\hat{C}_{n}^{\alpha_{1} \alpha_{2}} j_{\alpha_{1}} j_{\alpha_{2}}, \\
& l_{q}=\hat{C}_{q}^{\alpha_{1} \alpha_{2} \alpha_{3}} j_{\alpha_{1}} j_{\alpha_{2}} j_{\alpha_{3}}, \\
& \bar{l}_{w}=\hat{\bar{C}}_{w}^{\alpha_{1} \alpha_{2} \alpha_{3}} j_{\alpha_{1}} j_{\alpha_{2}} j_{\alpha_{3}},
\end{aligned}
$$

and if these expressions are substituted into (3.17), then from (3.17) and (3.16) we obtain linear relations between the corresponding expansion coefficients by appropriate projections. This yields the relations

$$
\begin{aligned}
& \left.\left\langle D_{n}\left(\beta_{1} \ldots \beta_{n}\right) \mathfrak{F}\right\rangle=\varphi_{n}\left(\beta_{1} \ldots \beta_{n}\right) \mid a\right) \\
& =\sum_{N . K, L} c\left(n_{1} \ldots n_{N}, q_{1} \ldots q_{K}, w_{1} \ldots w_{L} \mid a\right) \\
& \quad \cdot\left\langle D_{n}\left(\beta_{1} \ldots \beta_{n}\right)\left|b_{n_{1}} \ldots b_{n_{N}}, l_{q_{1}} \ldots l_{q_{K}}, \bar{l}_{w_{1}} \ldots \bar{l}_{w_{L}}\right| 0\right\rangle .
\end{aligned}
$$

[1] F. Bopp, Ann. Phys., Germany 38, 345 (1940).

[2] B. Podolski, Phys. Rev. 62, 68 (1942).

[3] K. Wildermuth, Z. Naturforsch. 5a, 373 (1950).

[4] H. Stumpf, Physica 114 A, 184 (1982).

[5] D. Grosser, Z. Naturforsch. 38 a, 1293 (1983).

[6] D. Grosser and T. Lauxmann, J. Phys. G 8, 1505 (1982).

[7] H. Stumpf, Z. Naturforsch. 37 a, 1295 (1982).

[8] D. Grosser, Z. Naturforsch. 38a, 1293 (1983).

[9] H. Stumpf, Z. Naturforsch. 39 a, 441 (1984).

[10] D. Grosser, B. Hailer, L. Hornung, T. Lauxmann, and H. Stumpf, Z. Naturforsch. 38 a, 1056 (1983).

[11] H. Stumpf, Z. Naturforsch. 38a, 1064 (1983); 38 a, 1184 (1983).

[12] L. de Broglie, C.R. Acad. Sci. Paris 195, 862 (1932); 199, 813 (1934).
One can also try to establish the reverse relation, i.e. to express the $C(N, K, L)$ in terms of the $\varphi_{n}$ by using the representations (3.14), (3.15a), (3.15b). However, we do not perform this explicitly because in any case both kinds of relations are, due to their complexity, of no use for practical calculations, and such calculations are not our intention. We are rather satisfied by having demonstrated that an expansion of the kind (3.17) is possible. The next step in our program is to transform the functional equation (1.15) from the elementary fermion source representation into a cluster source representation and to calculate the functional state (3.17) directly by means of this transformed equation. In this way the use of the complicated relations (3.20) ect. is avoided.

Finally, we consider the symmetric properties of the expansion (3.17). By direct calculation it follows from (3.8), (3.9a), (3.9b) that the following relations hold

$\left[b_{n}, b_{n^{\prime}}\right]_{-}=0 ; \quad\left[l_{q}, l_{q^{\prime}}\right]_{+}=0 ; \quad\left[\bar{l}_{w}, \bar{l}_{w^{\prime}}\right]_{+}=0$.

Therefore, $c\left(n_{1} \ldots n_{N}, q_{1} \ldots q_{K}, w_{1} \ldots w_{L}\right)$ must be symmetric with respect to permutations of $n_{1} \ldots n_{L}$ and antisymmetric with respect to permutations of $q_{1} \ldots q_{K}$ and $w_{1} \ldots w_{L}$ respectively. This is of course the influence of the Pauli principle with respect to the clusters as a whole. The expansion (3.17) does, however, not contain the exchange of fermions between different clusters as was emphasized above.

\section{Acknowledgement}

I would like to thank Dr. D. Grosser and Prof. Dr. P. Kramer for a critical discussion of the manuscript.

[13] E. Fermi and C. N. Yang, Phys. Rev. 76, 1739 (1949).

[14] W. Heisenberg, Introduction to the Unified Field Theory of Elementary Particles, Interrsc. Publ., London 1966.

[15] B. Jouvet, Suppl. Nuovo Cim. 2, 941 (1955); Nuovo Cim. 3, 1133 (1956); 5, 1 (1957).

[16] R. Haag, Phys. Rev. 112, 669 (1958).

[17] K. Nishijima, Phys. Rev. 111, 995 (1958).

[18] W. Zimmermann, Nuovo Cim. 10, 597 (1958).

[19] J. D. Bjorken, Ann. Phys. (N.Y.) 24, 174 (1963).

[20] I. Bialynicki-Birula, Phys. Rev. 130, 465 (1963).

[21] D. Lurie and A. J. Macfarlane, Phys. Rev. 136, B 816 (1964).

[22] G. S. Guralnik, Phys. Rev. 136, B 1404 (1964).

[23] R. L. Zimmermann, Phys. Rev. 141, 1554 (1966); 146, 955 (1966); 164, 1945 (1967). 
[24] B. Jouvet, Solid State, Nuclear Physics and Particle Physics; 9th Latin American School of Physics, Santiago (Chile 1967), ed. I. Saavedra, W. A. Benjamin, New York 1968, pp. 89-255.

[25] J. Mandelbrojt, Ann. Inst. H. Poincaré A 7, 257 (1967).

[26] V. Massida and E. Tirapegui, Ann. Inst. H. Poincaré A 11, 439 (1969).

[27] F. Esposita and U. Esposito, Nuovo Cim. 67A, 377 (1970).

[28] E. Tirapegui, Nucl. Phys. B 34, 598 (1971).

[29] K. G. Wilson and W. Zimmermann, Commun. Math Phys. 24, 87 (1972).

[30] P. Otterson and W. Zimmermann, Commun. Math. Phys. 24, 107 (1972).

[31] P. Yuk-Ming Lam and B. Schroer, Phys. Rev. D 8, 657 (1973)

[32] S. Coleman, R. Jackiw, and H. D. Politzer, Phys. Rev. D 10, 2491 (1974)

[33] D. J. Gross and A. Neveu, Phys. Rev. D 10, 3235 (1974).

[34] T. Kugo, Prog. Theor. Phys. 55, 2032 (1976); Phys. Lett. B 76, 625 (1978).

[35] K. Kikkawa, Prog. Theor. Phys. 56, 947 (1976).

[36] T. Eguchi, Phys. Rev. D 14, 2755 (1976).

[37] T. Saito and K. Shigemoto, Prog. Theor. Phys. 57, 242 (1977); 57,643 (1977).

[38] H. Terazawa, Y. Chikashige, and K. Akama, Phys. Rev. D 15, 480 (1977).

[39] G. Konisi, H. Miyata, T. Saito, and K. Shigemoto, Prog. Theor. Phys. 57, 2116 (1977).

[40] K. Tamvakis and G. S. Guralnik, Phys. Rev. D 18, 4551 (1978).

[41] D. Campbell, F. Cooper, G. S. Guralnik, and N. Snyderman, Phys. Rev. D 19, 549 (1979).

[42] R. W. Haymaker and F. Cooper, Phys. Rev. D 19, 562 (1979).

[43] F. Cooper, G. S. Guralnik, R. W. Haymaker, and K. Tamvakis, Phys. Rev. D 20, 3336 (1979).

[44] K. Shizuya, Phys. Rev. D 21, 2327 (1980).

[45] H. J. Munczek and A. M. Nemirovsky, Phys. Rev. D 22, 2001 (1980).

[46] P. Rembiesa, Phys. Rev. D 24, 1647 (1981).

[47] M. Konisi and W. Takahasi, Phys. Rev. D 23, 380 (1981).

[48] H. S. Sharatchandra, Phys. Rev. D 23, 3025 (1981).

[49] C. C. Chiang, C. B. Chiu, E. C. G. Sudarshan, and X. Tata, Phys. Rev. D 25, 1136 (1982).

[50] H. J. Munczek, Phys. Rev. D 25, 1579 (1982).

[51] S. P. Chia, C. B. Chiu, and K. C. Chou, Phys. Lett. B 109, 457 (1982)

[52] M. M. Broido, Phys. Rev. 157, 1444 (1967).

[53] M. La Camera and A. Wataghin, Lett. Nuovo Cim. 4, $693(1970)$.

[54] W. Kerler, Phys. Lett. B 69, 355 (1977).

[55] W. Kerler, Nucl. Phys. B 202, 437 (1982).

[56] T. Eguchi, Phys. Rev. D 17,611 (1978).

[57] K. Tamvakis and G. S. Guralnik, Nucl. Phys. B 146, 224 (1978).

[58] T. Banks and A. Zaks, Nucl. Phys. B 184, 303 (1981).

[59] C. C. Chiang, C. B. Chiu, E. C. G. Sudarshan, and X. Tata, Physica 114A, 176 (1982).

[60] N. Nakanishi, Prog. Theor. Phys. 68, 287 (1982).

[61] U. Ellwanger, Nucl. Phys. B 207, 447 (1982).

[62] H. P. Dürr and H. Saller, Phys. Rev. D 22, 1176 (1980); H. P. Dürr, Nuovo Cim. 62 A, 69 (1981); 73 A,
165 (1983); Phys. Lett. B 115, 33 (1982); B 122, 368 (1983).

[63] H. Saller, Nuovo Cim. 67A, 70 (1982); 68 A, 324 (1982); 71 A, 17 (1982).

[64] D. Amati and G. Veneziano, Nucl. Phys. B 204, 451 (1982).

[65] J. A. Wheeler, Phys. Rev. 52, 1107 (1937); 52, 1083 (1937).

[66] J. J. Griffin and J. A. Wheeler, Phys. Rev. 108, 311 (1957).

[67] H. Ekstein, Phys. Rev. 101, 880 (1956).

[68] K. Wildermuth and T. Kannelopoulos, Nucl. Phys. 7 , 150 (1958).

[69] K. Wildermuth and Y. C. Tang, A Unified Theory of the Nucleus, Vieweg, Braunschweig 1977.

[70] T. F. Jordan, J. Math. Phys. 5, 1345 (1964).

[71] F. Koutchouk, Nuovo Cim. 57 A, 483 (1970).

[72] M. D. Girardeau, Phys. Rev. Lett. 27, 1416 (1971); J. Math. Phys. 16, 1901 (1975); 19, 2605 (1978).

[73] L. Rosenberg, Phys. Rev. D 9, 1789 (1974).

[74] A. Goldenberg and R. D. Puff, Phys. Rev. A 10, 323 (1974).

[75] J. D. Gilbert, J. Math. Phys. 18, 791 (1977).

[76] V. Kvasnicka, Czech. J. Phys. B 32, 947 (1982).

[77] P. Kramer, G. John, and D. Schenzle, Group Theory and the Interaction of Composite Nucleon Systems, Vieweg, Braunschweig 1981.

[78] E. W. Schmid, Phys. Rev. C 21, 691 (1980). N-Cluster dynamics and effective interactions of composite particles II, preprint Univ. Tübingen 1980.

[79] H. Stumpf, Acta Phys. Austriaca Suppl. 9, 195 (1972).

[80] H. Stumpf, Z. Naturforsch. 36 a, 1289 (1981).

[81] S. Tomonaga, Prog. Theor. Phys. 1, 27 (1946).

[82] J. Schwinger, Phys. Rev. 74, 1439 (1948).

[83] F. Bopp, Z. Physik 171, 90 (1963).

[84] C. S. Lam, Nuovo Cim. 59 A, 435 (1969).

[85] F. R. Halpern, J. Math. Phys. 8, 648 (1967).

[86] A. L. Licht and A. Pagnamento, Phys. Rev. D 2, 1150 (1970); 2, 1156 (1970).

[87] A. Peres, Nuovo Cim. 10 A, 230 (1972).

[88] D. Lurie, Particles and Fields. Interscience Publ. New York 1968.

[89] R. Hagedorn, Fortschr. Physik, Sonderband 5, Akademie Verlag, Berlin 1963.

[90] B. Hailer, Thesis, Universität of Tübingen, in preparation.

[91] Y. Kazama and York-Peng Yao, Phys. Rev. D 21 , 1116 (1980); 21, 1138 (1980); 25, 1605 (1982).

[92] T. Appelquist and J. Carrazone, Phys. Rev. D 11, 2856 (1975).

[93] B. Ovrut and H. J. Schnitzer, Phys. Rev. D 21, 3369 (1980); 22, 2518 (1980); 24, 1695 (1981).

[94] W. I. Weisenberger, Phys. Rev. D 24, 481 (1981); 24, $1617(1981)$

[95] C. Lee and P. Y. Pac, Nucl. Phys. B 185, 485 (1981).

[96] E. B. Manoukian, J. Math. Phys. 22, 572 (1981); 22, 2258 (1981).

[97] N. P. Chang, A. Das, D. X. Li, D. Ch. Xian, and X. J. Zhou, Phys. Rev. D 25, 1630 (1982).

[98] R. Akhoury and Y. P. Yao, Phys. Rev. D 25, 3361 (1982).

[99] G. McKeon, Phys. Rev. D 26, 2086 (1982).

[100] R. Barbieri, S. Ferrara, and D. V. Nanapoulos, Phys. Lett. B 116, 16 (1982). 